Mots. Les langages du politique

78 | 2005

Usages politiques du genre

\title{
Ethos de femmes ministres. Recherche d'indices quantifiables
}

Serge Vassy

\section{OpenEdition}

Journals

Édition électronique

URL : https://journals.openedition.org/mots/422

DOI : $10.4000 /$ mots.422

ISSN : 1960-6001

Éditeur

ENS Éditions

Édition imprimée

Date de publication : 1 juillet 2005

Pagination : 105-114

ISBN : 2-84788-080-1

ISSN : 0243-6450

Référence électronique

Serge Vassy, «Ethos de femmes ministres. Recherche d'indices quantifiables », Mots. Les langages du politique [En ligne], 78 | 2005, mis en ligne le 31 janvier 2008, consulté le 24 avril 2022. URL : http:// journals.openedition.org/mots/422 ; DOI : https://doi.org/10.4000/mots.422 


\section{Ethos de femmes ministres. Recherche d'indices quantifiables}

Au cours de ces dix dernières années, avec des temps forts que nourrissent les échéances électorales et l'actualité politique, s'est développé en France un débat public sur le rapport des femmes à la politique, et plus particulièrement sur la présence féminine dans les diverses instances du pouvoir (partis, parlement, gouvernement.... ${ }^{1}$.

Parallèlement aux discussions sur la nécessité de promouvoir l'équité femme/homme dans ce champ social majeur, s'est affirmé - porté par une médiatisation relativement prolifique - un modèle valorisant de la femme politique, en contraste avec les représentations de leurs pairs masculins. Au travers de leur engagement et de leur action, les femmes actualiseraient des qualités telles que le sens des responsabilités, du travail et du concret, le goût de la précision et de la nuance, le refus de la polémique, l'attachement au collectif et l'absence d'ambitions personnelles, la capacité à prendre davantage en compte la dimension « humaine » des dossiers, etc.

Dans ce contexte, quelques rares travaux ont abordé la problématique de la sexuation du langage, dans la perspective de la communication textuelle politique et publique. Mobilisant différentes méthodologies, ces études tentent d'appréhender le comportement verbal des personnalités politiques féminines, parmi lesquelles des ministres s'exprimant dans le cadre de l'exercice du pouvoir.

Notre travail s'inscrit dans le prolongement de ces recherches, avec le souci de dépasser un écueil méthodologique fondamental : l'analyse qualitative de gros corpus contraint le chercheur à y sélectionner des textes relativement courts. Ce prélèvement d'échantillons comporte un risque, celui d'opérer des cadrages en harmonie avec les représentations sociales dominantes, voire avec les hypothèses initiales de l'étude.

1. Par exemple: Édith Cresson, Premier ministre de François Mitterrand; les «jupettes», ministres évincées du premier gouvernement Juppé; les femmes ministres du gouvernement Jospin, chargées de portefeuilles importants dans un contexte de débat sur la parité; Michèle Alliot-Marie, ministre de la Défense du gouvernement Raffarin...

Université Paris 12, CEDITEC, svassy@wanadoo.fr 


\section{Antécédents et objectifs du travail de recherche}

\section{La communication verbale du premier gouvernement Jospin}

En 2000, une équipe pluridisciplinaire a mené une triple analyse d'un corpus multigenre constitué par les interventions de huit ministres, dont L. Jospin, dans les médias et à l'Assemblée ${ }^{2}$. Trois méthodologies ont été mobilisées: analyse de contenu, utilisée par les sciences de l'information et de la communication, lexicométrie et analyse énonciative-argumentative, utilisées par les sciences du langage.

L'analyse énonciative et argumentative portait sur un ensemble de textes caractérisés par des thèmes plus ou moins transversaux (emploi et insertion des jeunes, exclusion, violence, sécurité), dossiers socio-économiques prioritaires pour l'action des ministres.

Différents constats et hypothèses avaient été formulés, dont l'existence de trois "ethos discursifs " $^{3}$ permettant le regroupement des ministres (quatre femmes et quatre hommes) par delà les clivages politiques, les domaines de compétence et les thématiques abordées : le modèle "pondéré » (Lionel Jospin et Dominique Strauss-Kahn), le modèle «polémique» (Claude Allègre et Jean-Pierre Chevènement) et celui qui nous intéresse ici, le modèle «pragmatique empathique» (Martine Aubry, Marie-George Buffet, Elisabeth Guigou et Dominique Voynet).

Ce dernier modèle se fondait sur l'observation de traits langagiers spécifiques caractérisant la présentation de soi des ministres femmes, deux socialistes, une écologiste et une communiste. Le pragmatisme, l'affichage d'une connaissance approfondie des dossiers, la volonté d'éviter les lieux communs et les généralisations abusives, etc. Les quatre ministres femmes partageaient un discours du sérieux et de la rigueur, mais leur parole était aussi celle du « vécu » et du « senti ». Observation plutôt inattendue, le modèle «pragmatique empathique» apparaissait donc comme un possible "ethos féminin » du discours ministériel, caractérisé par un mixte de concret, d’émotion et d'empathie.

2. S. Bonnafous et S. Vassy, 2001, «Réflexions sur une étude de la communication gouvernementale», Émergence et continuité dans les recherches en information et communication, Actes du $12^{\mathrm{e}}$ congrès national de la Société française des sciences de l'information et de la communication, 10-13 janvier 2001, Paris, SFCIC, p. 205-211.

3. Tout énonciateur peut utiliser trois sortes de preuves pour soutenir son argumentation (Aristote, livre 1 de la Rhétorique) : celles qui concernent son caractère (ethos), celles qui visent la réceptivité de l'auditoire (pathos) et enfin, les arguments eux-mêmes (logos). La notion d'ethos discursif englobe donc tout ce qui, dans la parole d'un énonciateur, fait émerger, sous son contrôle et à son insu, une représentation de sa personne dans l'auditoire. 


\section{Singularisations discursives des femmes politiques}

Partant de ces observations, S. Bonnafous 4 a étudié la manifestation du genre dans les prises de parole de différentes femmes investies de responsabilités politiques, au sens large. Pour ce faire, elle a élargi le corpus des ministres à d'autres femmes politiques de gauche et de droite, à des responsables syndicales et à des personnalités étrangères.

Ce travail distingue trois modes d'expression discursive du genre.

Avec l'argument explicite par le genre5, la locutrice convoque sa qualité de femme en appui de la thèse soutenue. Faiblement attesté, ce type d'argumentation sert surtout à souligner les discriminations dont les femmes politiques s'estiment victimes.

Opérant comme un véritable retournement de stigmate, l'argumentation implicite par le genre consiste en l'utilisation stratégique de qualités stéréotypiques féminines, qui se présentent comme un atout en matière d'action politique. Parmi les plus fréquemment évoquées, on distingue le sérieux, le goût du concret, l'habitude du travail, la sensibilité, le dévouement, la simplicité.

Le troisième mode serait une manière plus ou moins consciente de se présenter, au moyen d'éléments lexicaux, énonciatifs et argumentatifs, c'est-àdire la mobilisation non maîtrisée d'un ethos discursif féminin.

\section{Politique et ethos féminin : recherche d'indices quantifiables de la sexuation discursive}

Notre travail partage avec le précédent le même fil d'Ariane : à partir de l'hypothèse d'un ethos discursif féminin en politique, il s'agit d'essayer d'appréhender et de décrire les aspects qui, dans la communication verbale des femmes, apparaissent comme des spécificités. Ces singularisations langagières résulteraient d'un jeu complexe d'au moins deux facteurs, l'incorporation par les femmes d'un modèle socialement normé et la mobilisation stratégique, dans la communication, de qualités stéréotypiques valorisantes.

Lors des deux précédents travaux, l'exploration des corpus au moyen d'une lecture cursive privilégiait l'évaluation qualitative de phénomènes langagiers focalisés. Notre travail fait appel à la lexicométrie, méthodologie quantitative qui procède par délinéarisation du texte et permet de repérer des

4. S. Bonnafous, 2003, "“Femme politique”, une question de genre?», Réseaux, n 120, p. 119145 .

5. Proposé par A. Krieg-Planque à partir de l'étude des interviews de femmes politiques publiées dans Le Monde, entre 1995 et 2000. Dans un ensemble de 126 textes, quatre argumentations de ce type ont été repérées. 
faits langagiers diffus. Ceci entraîne un déplacement du point de vue, rendant de ce fait l'observation moins sensible aux a priori.

\section{Le corpus artificiel dense}

Le corpus sur lequel nous travaillons partage avec celui de l'étude sur la communication verbale du premier gouvernement Jospin des caractéristiques fondamentales. Les productions textuelles considérées sont celles des mêmes huit ministres: quatre femmes et quatre hommes issus, rappelons-le, des diverses composantes de la «majorité plurielle», PS, PCF, Verts, Mouvement des citoyens. Il s'agit de prises de parole dans les médias (presse écrite et audiovisuelle), de juillet 1997 à décembre 1999.

Deux particularités majeures doivent toutefois être soulignées.

La première est une originalité méthodologique qui soulève des questions épistémologiques : nous utilisons la lexicométrie sur des textes non intégraux. Qu'il s'agisse d'entretiens, de tribunes libres ou d'interventions à l'Assemblée, nous n'avons pas gardé in extenso les textes - tous polythématiques mais en avons sélectionné des segments monothématiques. Nous travaillons sur une concaténation d'extraits d'interventions ministérielles, sélectionnés sur la base d'une double analyse sémantique et lexicale, d'où l'appellation de «corpus artificiel dense ${ }^{6}$. On sait l'analyse lexicométrique très sensible à la thématique des textes. L'abandon de la prise de parole comme unité textuelle de base produit une densification thématique du corpus. Celle-ci renforce sa cohérence, limitant le nombre de variables et rendant donc l'interprétation des résultats plus robuste.

Deuxième particularité, les thèmes des extraits que nous avons retenus ne sont plus ceux de l'étude sur la communication du premier gouvernement Jospin, «emploi et insertion des jeunes», «exclusion», "violence» et "sécurité ». Nous travaillons maintenant sur «majorité plurielle», c'est-à-dire le travail de l'équipe gouvernementale et de sa majorité, et sur «conception de la politique », l'analyse de l'action à la lumière de principes et valeurs qui fondent l'engagement personnel. Ainsi, aux thèmes socio-économiques prioritaires de l'action ministérielle, nous avons substitué un métadiscours caractéristique du gouvernement Jospin, lequel a consacré une part importante de son expression publique à expliciter sa conception de la politique. Le nouveau corpus répond mieux à notre souhait de constituer un ensemble de textes

6. Afin de rendre la sélection la plus stable et objective possible, nous avons utilisé des grilles d'analyse. La présence de certains lexèmes (majorité, gouvernement...), la référence à un courant de pensée, à des valeurs... nous ont permis de décider qu'un passage d'un texte correspondait aux thèmes étudiés. 
davantage marqué par la transversalité, afin de renforcer la pertinence des comparaisons. Nous en profitons pour vérifier un éventuel effet des variations thématiques sur la parole des gouvernants.

Le corpus ainsi constitué comprend un peu plus de 31600 formes pour environ 251800 occurrences ${ }^{7}$. Les prises de parole des ministres femmes représentent 106800 occurrences, contre 145000 pour les ministres hommes, soit une sous-représentation d'environ $25 \%$. La parole ministérielle se répartit de manière équivalente entre médias audiovisuels (télévision $27 \%$, radio $23 \%$ ) et presse écrite (quotidiens $24 \%$, hebdomadaires $19 \%$, mensuels $7 \%$ ). Quant aux genres médiatiques, l'entretien prédomine largement, avec 208000 occurrences, soit $83 \%$ du corpus. Les entretiens télévision et radio, assez nombreux, produisent une parole relativement spontanée et moins contrôlée par le locuteur, ce qui est intéressant pour notre étude ${ }^{8}$.

\section{Méthodologies et premiers résultats}

\section{Une exploration artisanale}

Tout corpus soumis au logiciel Lexico $3^{9}$ est segmenté en formes graphiques. Une liste exhaustive de tous les lexèmes présents est générée, que l'on peut classer par ordre lexicographique (alphabétique) ou lexicométrique (par fréquence ou nombre d'occurrences de chaque forme). Partant d'un classement lexicométrique descendant, nous avons assigné un rang à chaque lexème: le rang 1 est attribué à la forme la plus fréquente du corpus puis, plus la fréquence diminue, plus le rang augmente. Fréquence et rang sont donc en rapport inversé.

Nous avons observé les cent premières formes les plus fréquentes dans la totalité du corpus et chez chaque ministre. Ces listes de mots vides et pleins,

7. Du point de vue lexicométrique, une forme est un lexème caractérisé par sa morphologie, c'est-à-dire une unité de langue. On nomme occurrence chaque apparition d'une forme dans un corpus.

8. Si la communication des ministres à la télévision et à la radio impose la présence physique du locuteur (entretien, débat...), il en va tout autrement dans la presse écrite : les textes sont souvent rédigés par des "écrivants », acteurs importants des cabinets ministériels, majoritairement hommes. La question peut donc légitimement être posée: qui, de la femme ministre ou de l'homme "écrivant», s'exprime dans ces prises de parole? L'enquête de C. Ollivier-Yaniv sur les conditions de production de la communication ministérielle souligne l'intervention active des femmes ministres dans la préparation des textes (relecture, corrections, validations, réécriture...) et la mimétisation des «écrivants », qui finissent par incorporer les spécificités verbales du ministre pour lequel ils travaillent. C. Ollivier-Yaniv, 2003, "La fabrique du discours politique : les “écrivants" des prises de parole ministérielles ", Rhétorique et discours politique, Actes du colloque de Cerisy, Presses universitaires de Rennes, p. 89-98.

9. Lexico 3 a été développé par C. Lammalle, W. Martinez et A. Salem. 


\section{SERge VASSY}

paradigme lexical du discours ministériel, fournissent des informations intéressantes. Les noms constituent l'une des composantes du vocabulaire ministériel partagé ${ }^{10}$ : politique, gouvernement, aujourd'hui, gauche, France, majorité, pays, Français, Europe, ministre et État (classés par ordre décroissant de fréquence). Il en est de même des pronoms, des verbes et des adverbes, des connecteurs logiques... Par induction, commencent à émerger de ce travail exploratoire des pistes de recherche.

On peut observer les cent premières formes utilisées par chaque ministre et réaliser une analyse contrastive. Au niveau des noms communs, les spécificités de chaque locuteur actualisent des déterminations stylistiques, politiques, gouvernementales, d'agenda... Par exemple, chez C. Allègre : choses, éducation, temps, problème, école, idées et monde partagent les premiers rangs avec certains noms de la liste générale; chez D. Voynet, Verts, travail, façon, environnement, temps, parti et nucléaire font de même.

Quant aux marques de la sexuation du discours politique féminin, aucune singularité franche n'apparaît ici. Soulignons cependant la présence de femmes, hommes, vie, rôle et envie, parmi les cent premières formes de M.-G. Buffet. Chez l'une ou plusieurs des trois autres ministres femmes, on trouve responsabilité, travail, personnes et façons. Il s'agit d'indices intéressants, qui renvoient à certaines observations effectuées lors des précédentes recherches et qu'il convient d'explorer par un retour au texte.

Le rang d'une forme donnée peut être une autre entrée dans la recherche de marques langagières du genre. La pronominalisation, vecteur de l'implication discursive, en fournit un exemple. Dans le corpus dense total, le je occupe le rang moyen 13. Le rang du pronom chez chacun des ministres met en évidence une dispersion relativement importante, signe d'embrayages énonciatifs distincts : Buffet et Allègre, $r=9$; Aubry, Voynet et Jospin, $r=11$; Guigou, $r=13$; Chevènement, $r=17$ et Strauss-Kahn, $r=26$. Le constat est aisé, les quatre ministres femmes ont un emploi plutôt fréquent du je, ainsi que deux ministres hommes.

La notion de rang médian, c'est-à-dire la valeur qui scinde les huit ministres en deux groupes de même nombre est en bonne adéquation avec la problématique de la sexuation langagière. Toute forme faisant apparaître une bipolarisation femmes/hommes aurait un emploi nécessairement fortement corrélé avec le genre des locuteurs.

Observons, par exemple, la personne indéterminée on, vecteur de la prise de distance énonciative. La distribution autour du rang médian virtuel 24,5 est la suivante:

$r<24,5$ Aubry, Guigou et Strauss-Kahn (20), Buffet (24)

$r$ > 24,5 Allègre (25), Voynet (26), Chevènement (28), Jospin (33)

10. Plus précisément, l'un des territoires thématiques de ce type de discours. 
La mixité des groupes ne semble pas mettre en évidence une corrélation forte de l'emploi du on et du genre des énonciateurs. Mais la présence d'Aubry, Guigou et Buffet dans le groupe des fréquences élevées, ainsi que le rang du on chez Voynet, légèrement supérieur au rang médian, indiquent une tendance au suremploi de ce pronom chez les ministres femmes.

Nous avons utilisé cette méthode pour analyser la pronominalisation, la négativité, les adverbes de quantité et d'intensité, la forme interrogative. Résultat prévisible, ces tests ne révèlent pas de clivage net et systématique femmes/hommes. Par contre, à plusieurs reprises, la proximité relative des femmes nous incite à penser la sexuation discursive en termes de tendance.

\section{L'analyse lexicométrique}

Lexico 3 est un outil assez puissant de comptage et de calcul, qui laisse la responsabilité de l'analyse et de l'interprétation des résultats au chercheur. Nous avons donc utilisé cette application pour explorer plus en profondeur et plus précisément le corpus dens $\mathrm{e}^{11}$. Évoquons brièvement quelques résultats.

Nous avons étudié l'emploi du groupe [femme (24), femmes (103) $]^{12}$. Un nombre relativement important d'occurrences caractérise les quatre femmes, ainsi que L. Jospin. En revanche, les autres ministres, trois hommes, emploient peu les deux noms communs. Si l'on considère le résultat probabilisé, seules Buffet (12) et Aubry (2) suremploient femme(s).

L'observation du groupe [homme (96), hommes (100)] met en évidence un suremploi chez Buffet, Aubry et Jospin. Un retour au texte pour observer les contextes de femme(s) révèle un nombre important de cooccurrences $(42,5 \%)$. On peut, bien sûr, penser à certains quasi-figements caractéristiques du discours politique (les femmes et les hommes de ce pays...). Mais cette proportion élevée de cooccurrences femme(s)-homme(s) renvoie à une volonté discursive, celle de ne pas se limiter au générique masculin. On trouve là une mise en parole de la parité, thème très présent dans l'expression et l'action du gouvernement Jospin, terrain de différenciation politique par rapport à la majorité précédente et au président de la République.

Dans la perspective de caractériser les régimes énonciatifs individuels, nous avons observé l'emploi des pronoms et adjectifs de première personne du singulier (je, j', moi, me, m', ma, mon, mes), ceux de première personne du pluriel (nous, notre, nos) et la personne indéterminée on. Nous n'avons pas

11. Nous avons utilisé le calcul des spécificités de certains formes (femme), groupes de formes (femme, femmes, féminin, féminité) et segments répétés (les femmes et les hommes). Les spécificités sont des calculs probabilistes qui tiennent compte du nombre d'occurrences total et de celui du locuteur considéré. Des spécificités positives indiquent un suremploi des formes concernées et vice versa.

12. Entre parenthèses, le nombre total d'occurrences de la forme dans le corpus total. 
constaté de clivage franc femmes/hommes. Mais, en tendance, les femmes se singularisent par un régime énonciatif en $\mathrm{je}^{+} /$nous $^{-} / \mathrm{on}^{+}$. Le paradoxe de l'implication discursive moyenne/forte en $j e^{+}$et du désengagement en ${ }^{+}{ }^{+}$peut sans doute s'expliquer par l'utilisation du on en remplacement du nous, dans une démarche de proximité et de simplicité langagière. Bien que caractéristique du genre de l'entretien dans les médias audiovisuels, le suremploi du on révèlerait donc ici un comportement énonciatif féminin spécifique.

Nous avons observé la modalisation discursive en étudiant l'emploi des adverbes de quantité (assez, beaucoup, moins, peu, très, trop) et d'intensité terminés en ment (une trentaine présents dans le corpus). Un suremploi chez les femmes aurait pu caractériser un discours du « senti », renvoyer à une forme de véhémence lexicale. Or, les résultats obtenus montrent que cet indice n'est pas discriminant.

Nous nous sommes intéressé aux verbes déclaratifs croire, penser, dire, considérer et trouver, assez fréquents dans le discours politique. Seul le verbe croire est relativement discriminant: on constate une utilisation moyenne-forte chez les femmes. Contrairement à penser et considérer, croire renvoie à un engagement fort, sur la base de certitudes qui n'ont pas forcément besoin d'être démontrées. Mais en même temps, croire pourrait indiquer une prudence argumentative (je crois que...), une manière moins conflictuelle d'envisager le rapport au contradicteur et à l'adversaire. Ce qui peut faire penser à la moindre agressivité et à l'évitement des situations polémiques chez les femmes politiques, pointés par S. Bonnafous, et aux observations de certains sociolinguistes : les femmes seraient moins assertives que les hommes, préférant un mode plus coopératif que compétitif.

L'étude des verbes et locution verbale de la volonté et du souhait, vouloir, espérer, souhaiter et avoir envie révèle des résultats contrastés. D’une part, l'emploi du groupe vouloir ${ }^{13}$ ne discrimine pas les femmes et les hommes. Seul le Premier ministre se distingue par un suremploi marqué, ce qui renvoie sans doute à son rôle au sein de l'équipe gouvernementale - hypothèse confirmée par l'étude du syntagme je veux, que L. Jospin est le seul des huit locuteurs à utiliser relativement fréquemment.

D'autre part, les ministres femmes se distinguent par un suremploi de avoir envie et souhaiter, avec prédominance de l'un ou l'autre suivant la locutrice considérée. Trois des quatre femmes suremploient envie (conjugaison de la locution verbale avoir envie et le substantif), alors que la quatrième fait de même avec le groupe souhaiter ${ }^{14}$. Ce phénomène est d'autant plus intéressant

13. Vouloir (37), veux (69), voudrais (65), voulais (10), voulons (54), voulions (8).

14. Souhaite (74), souhaiterai (1), souhaiterais (3), souhaitais (1), souhaitons (9), souhaitions (1), souhaitait (4), souhaitant (1). 
que les quatre locuteurs hommes affichent une utilisation banale ou spécifique négative, quels que soient les verbes considérés.

Les contextes mettent en scène une diversité de sujets: la locutrice ellemême, «ce pays », « les gens », « les Français », " les femmes », « les ministres »...

[...] et moi c'est ça qui me fait vivre et qui fait que j'ai envie de me battre en politique. (M. Aubry)

Donnons à chacune et à chacun l'envie de se mêler de la chose publique.

(M.-G. Buffet)

J'ai envie de convaincre, j'ai envie de travailler, de dialoguer. (D. Voynet)

Moi, franchement, je souhaite qu'on ait une droite démocratique et républicaine. (E. Guigou)

Nous sommes devant un phénomène langagier intéressant car peu fréquent dans le discours politique: à des degrés divers, ces verbes font entendre la dimension du désir. Contrairement à la volonté, origine de l'action délibérée en vue d'atteindre une fin, le souhait pourrait suggérer une certaine passivité. En réalité, ici, le substantif envie évoque une tension essentielle, source de détermination à agir. Si les verbes de la volonté renvoient à la raison et à l'action immédiate, les verbes du souhait, et notamment la locution verbale avoir envie, expriment le ressenti, manifestent une pulsion intime génératrice de vitalité.

Bien qu'inachevée, l'analyse lexicométrique ne révèle pas une bipolarisation langagière nette des locuteurs femmes et hommes. Autrement dit, leurs interventions ne mettent pas en évidence un rapport mécanique genre-parole, ce qui était assez prévisible. Même dans un corpus monothématique, les singularités observées résultent de l'interaction de facteurs multiples: le champ de compétences, le rapport portefeuille-agenda politique, l'appartenance partisane... et aussi des modalités expressives personnelles. Les résultats de certains tests nous incitent cependant à penser la sexuation du discours politique en termes de tendance. Bien entendu, la pertinence des indices quantifiables devra être confirmée par l'étude d'un plus grand échantillon de locuteurs.

Par exemple, les femmes ministres se distinguent par un emploi moyen-fort du nom commun femmes(s) tandis que leurs pairs masculins se caractérisent par un emploi moyen-faible. De plus, l'emploi des cooccurrences femmes(s)hommes(s) dessine deux démarches: d'une part, un équilibre entre l'emploi isolé et la cooccurrence ( $M$. Aubry et M.-G. Buffet), réaffirmation d'un attachement à la parité ${ }^{15}$; d'autre part, une très nette préférence pour l'emploi isolé

15. «Les hommes et les femmes qui ont porté ce gouvernement jugent son action au fur et à mesure des décisions qu'il prend. » M.-G. Buffet, L'Humanité, 2 octobre 1997. 
(E. Guigou et D. Voynet), dans une argumentation qui vise plus activement la promotion politique et sociale des femmes ${ }^{16}$. Quant au Premier ministre, son emploi de femme(s) est fréquent avec une claire prédominance des cooccurrences: L. Jospin avait fait de la parité et de la place des femmes en politique un thème central de sa communication.

Sur le plan énonciatif, les femmes ministres se distinguent par une implication forte $\left(j e^{+}\right)$et une simplicité langagière actualisée en un nombre élevé de substitutions on/nous. Quant à l'utilisation des verbes déclaratifs, le suremploi de croire pourrait indiquer une prudence argumentative et une approche moins conflictuelle des échanges. Enfin, elles mobilisent plus fréquemment que leurs pairs masculins les verbes de souhait (notamment la locution verbale avoir envie), mise en parole d'une action gouvernementale et politique fondée sur des attentes personnelles fortes quant aux objectifs visés.

Le décalage relatif et provisoire entre nos observations et les résultats de l'étude énonciative-argumentative précédente est intéressant en lui-même. Le changement de corpus pourrait mettre au jour une spécificité du comportement verbal des ministres femmes: certains thèmes, par exemple les problèmes sociaux, qui influencent la vie des gens de manière relativement directe, pourraient constituer un terrain plus favorable à la manifestation de l'ethos féminin. Ainsi, aux effets des variations de contextes, de support et de genre médiatiques sur la façon dont les femmes politiques font mention de leur féminité, soulignés par S. Bonnafous, il conviendrait d'ajouter les effets des variations thématiques. Cette parole multiforme, modulée ici en fonction des territoires thématiques investis, pourrait être elle-même une caractéristique de l'ethos politique féminin.

16. «Les femmes ont toujours une approche concrète parce que, quelles que soient leurs responsabilités dans la politique, l'entreprise, les syndicats, les professions libérales, elles doivent se préoccuper de la vie quotidienne. » E. Guigou, Paris Match, 26 juin 1997. 